\title{
Focusing on brain tumours and brain metastasis
}

\author{
This Focus issue highlights current research into the unique biology of brain tumours and brain \\ metastasis and how this research might improve therapy of these often devastating diseases.
}

\section{despite the challenges presented by brain tumours, progress is being made on many different fronts against these often devastating diseases}

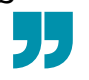

Survival for many types of malignant primary brain tumours has not improved much in the past 10 years, despite the introduction of some new treatments and despite our improved understanding of the biological bases of brain tumour development ${ }^{1,2}$. In addition, most malignant brain lesions are actually secondary brain tumours (brain metastases), and it is estimated that brain metastases will develop in up to $30 \%$ of adults who have a malignant primary tumour at another site $e^{2,3}$. Furthermore, brain tumours are the most common type of solid tumour in children and are the leading cause of cancer-related deaths in this population ${ }^{1,4}$. These statistics all indicate that better treatments for brain tumours and brain metastasis are a pressing need. We have therefore put together this Focus issue to highlight the diverse research in this field and the unique challenges posed by brain tumours and brain metastases.

Brain tumours are a heterogeneous group of diseases, but one important common feature is that they are subject to the unique biology of the brain and its microenvironment. The brain contains many cell types that are distinct from those found elsewhere in the body, making it challenging to extrapolate findings from cancers arising in other organs to those arising in the brain. Furthermore, the anatomy of the brain presents challenges for treating both brain tumours and brain metastases.

A prime example of both the unique biology and the anatomical challenges of treating brain tumours is the blood-brain barrier (BBB), the neurovascular unit that maintains brain homeostasis and acts as a 'gatekeeper', controlling the crossing of molecules and cells from the blood into the brain. Although the BBB is often disrupted in brain tumours, effective delivery of anticancer therapeutics through this blood-tumour barrier remains a challenge, as addressed by Arvanitis et al. ${ }^{5}$.

One aspect of the brain microenvironment that might not be as unique as initially presumed is the immune environment. The immune cell types of the brain differ from those in other organs, but, as discussed by Sampson et al. ${ }^{6}$, it is now becoming clear that this organ is not as 'immune privileged' as once thought, leading to hope that brain tumours and metastases might be successfully targeted with immunotherapies.
Gliomas account for $\sim 80 \%$ of malignant brain tumours, and the highest grade glioma, glioblastoma, is one of the most lethal cancers in adults ${ }^{3}$. Interestingly, genomic sequencing efforts more than 10 years ago jump-started the field of glioma metabolism with their finding of recurrent mutations in the genes encoding the tricarboxylic acid cycle enzymes IDH1 and IDH2, but the role of metabolism in glioma pathogenesis goes beyond IDH, as discussed by $\mathrm{Bi}$ et al. .

Medulloblastoma is one of the most common paediatric brain tumours ${ }^{4}$. Our understanding of this disease was advanced substantially by genomic studies reported in 2012. Since then, as discussed by Hovestadt et al. ${ }^{8}$, more genomic studies, as well as epigenomic, transcriptomic and proteomic profiling efforts, have provided new insights into medulloblastoma biology that will hopefully lead to improved diagnosis and therapy.

The prevalence of brain metastases in adults raises the question of what those working on primary brain tumours can learn from research on brain metastasis, and vice versa. This, and other important questions on brain metastasis, is pondered in a Viewpoint article written by four leading experts in this field ${ }^{9}$.

What has emerged from this collection of articles is that despite the challenges presented by brain tumours, progress is being made on many different fronts against these often devastating diseases, which will hopefully lead to improvements in survival in the next 10 years, if not sooner.

1. Jones, D. T. W. et al. Molecular characteristics and therapeutic vulnerabilities across paediatric solid tumours. Nat. Rev. Cancer 19, 420-438 (2019).

2. Lapointe, S. et al. Primary brain tumours in adults. Lancet 392 , 432-446 (2018).

3. Weller, M. et al. Glioma. Nat. Rev. Dis. Primers 1, 15017 (2015).

4. Liu, K. W. et al. Molecular mechanisms and therapeutic targets in pediatric brain tumors. Sci. Signal. 10, eaaf7593 (2017).

5. Arvanitis, C. D. et al. The blood-brain barrier and blood-tumour barrier in brain tumours and metastases. Nat. Rev. Cancer https://doi.org/10.1038/s41568-019-0205-x (2019).

6. Sampson, J. H. et al. Brain immunology and immunotherapy in brain tumours. Nat. Rev. Cancer https://doi.org/10.1038/ s41568-019-0224-7 (2019).

7. $\mathrm{Bi}$, J. et al. Altered cellular metabolism in gliomas - an emerging landscape of actionable co-dependency targets. Nat. Rev. Cancer https://doi.org/10.1038/s41568-019-0226-5 (2019).

8. Hovestadt, V. et al. Medulloblastomics revisited: biological and clinical insights from thousands of patients. Nat. Rev. Cancer https://doi.org/10.1038/s41568-019-0223-8 (2019).

9. Boire, A. et al. Brain metastasis. Nat. Rev. Cancer https://doi.org/ 10.1038/s41568-019-0220-y (2019). 